\begin{tabular}{|c|l|}
\hline Title & Total absolute horospherical curvature of submanifolds in hy perbolic space \\
\hline Author(s) & Buosi, Marcelo; Izumiya, Shyuichi; Soares Ruas, Maria A parecida \\
\hline Citation & Hokkaido University Preprint Series in Mathematics, 880, 1-16 \\
\hline Issue Date & 2007 \\
\hline DOI & 10.14943/84030 \\
\hline Doc URL & http://hdl.handle.net/2115/69689 \\
\hline Type & bulletin (article) \\
\hline File Information & pre880.pdf \\
\hline
\end{tabular}

Instructions for use 


\title{
TOTAL ABSOLUTE HOROSPHERICAL CURVATURE OF SUBMANIFOLDS IN HYPERBOLIC SPACE
}

\author{
MARCELO BUOSI, SHYUICHI IZUMIYA, AND MARIA APARECIDA SOARES RUAS
}

\begin{abstract}
We study the horospherical geometry of submanifolds in hyperbolic space. The main result is a formula for the total absolute horospherical curvature of $M$, which implies, for the horospherical geometry, the analogues of classical inequalities of the Euclidean Geometry. We prove the horospherical Chern-Lashof inequality for surfaces in 3-space and the horospherical Fenchel and Fary-Milnor's theorems.
\end{abstract}

\section{INTRODUCTION}

The hyperbolic Gauss map of a surface in hyperbolic space was introduced by Bryant [1] and Epstein [5] in the Poincaré ball model. Kobayashi[14, 15] has also independently defined it for a hypersurface in $H^{n}(\mathbb{R})=S O_{0}(n, 1) / S O_{n}$ under a different framework and studied some basic properties of it from the view point of the theory of Fourier transformations. In [6], the second named author, D. Pei and T. Sano define the hyperbolic Gauss-Kronecker curvature of hypersurfaces in hyperbolic space. Totally umbilic hypersurfaces in hyperbolic space are equidistant hypersurfaces, hyperspheres and hyperhorospheres which are the model hypersurfaces in hyperbolic space. The hyperbolic Gauss-Kronecker curvature is a hyperbolic invariant which describes the contact of hypersurfaces with such model hypersurfaces. The notion of horospherical Gauss-Kronecker curvature of hypersurfaces in the model of hyperbolic space in Minkowski space was introduced in [12]. This curvature is not a hyperbolic invariant but an $S O(n)$-invariant, where we consider the canonical $S O(n)$-subgroup in the group of hyperbolic motions. However it describes the contact of hypersurfaces with hyperhorospheres and it is independent of the choice of the model of hyperbolic space. We call the geometry related to this curvature the horospherical geometry of hypersurfaces in hyperbolic space ([6, 7, $9,10,13,12])$. Moreover, it has been shown in [12] that a Gauss-Bonnet type theorem holds when integration of this new curvature is taken over a closed orientable even-dimensional hypersurface.

On the other hand, the horospherical geometry of higher codimension submanifolds in hyperbolic space had been developed in [11]. In this paper we continue this investigation, with the purpose of studying global properties of such submanifolds. The main result is a formula for the total absolute horospherical curvature of $M$, which implies the analogues, for the horospherical geometry, of classical inequalities of the Euclidean geometry. We discuss the

2000 Mathematics Subject Classification. Primary 53A35; Secondary 53C99.

Key words and phrases. hyperbolic space, horospherical geometry, the Chern-Lashof type inequality.

The first author was partially supported by Conselho Nacional de Desenvolvimento Científico e Tecnológico (CNPq), Brazil, grant \# 141321/00-8. 
Chern-Lashof type inequality for surfaces in 3-space

$$
\int_{M}\left|\widetilde{\mathcal{K}}_{h}\right| d \mathfrak{v}_{M} \geqslant 2 \pi(4-\chi(M)) \text {. }
$$

This is a special feature of horospherical geometry. In fact, R. Langevin and G. Solanes in [17] contruct examples of surfaces in hyperbolic space which do not satisfy the Chern-Lashof type inequality, when the integral is taken with respect to the extrinsic curvature of the surface. In the last section we study curves in $H_{+}^{3}(-1)$, and obtain the horospherical analogues of the Fenchel, and the Fary-Milnor theorems.

\section{Notation AND DEFinitions}

We outline in this section the local differential geometry of hypersurfaces in the hyperbolic $n$-space developed in the previous papers $[6,12]$. We adopt, for this purpose, the model of hyperbolic $n$-space in Minkowski $(n+1)$-space. Let $\mathbb{R}^{n+1}=\left\{\left(x_{0}, x_{1}, \ldots, x_{n}\right) \mid x_{i} \in \mathbb{R}(i=\right.$ $0,1, \ldots, n)\}$ be an $(n+1)$-dimensional vector space. For any $\boldsymbol{x}=\left(x_{0}, x_{1}, \ldots, x_{n}\right), \boldsymbol{y}=$ $\left(y_{0}, y_{1}, \ldots, y_{n}\right) \in \mathbb{R}^{n+1}$, the pseudo scalar product of $\boldsymbol{x}$ and $\boldsymbol{y}$ is defined by $\langle\boldsymbol{x}, \boldsymbol{y}\rangle=-x_{0} y_{0}+$ $\sum_{i=1}^{n} x_{i} y_{i}$. We call $\left(\mathbb{R}^{n+1},\langle\rangle,\right)$ Minkowski $(n+1)$-space and denote it by $\mathbb{R}_{1}^{n+1}$. We say that a non-zero vector $\boldsymbol{x} \in \mathbb{R}_{1}^{n+1}$ is spacelike, lightlike or timelike if $\langle\boldsymbol{x}, \boldsymbol{x}\rangle>0,\langle\boldsymbol{x}, \boldsymbol{x}\rangle=0$ or $\langle\boldsymbol{x}, \boldsymbol{x}\rangle<0$ respectively. For a vector $\boldsymbol{v} \in \mathbb{R}_{1}^{n+1}$ and a real number $c$, we define the hyperplane with pseudo normal $\boldsymbol{v}$ by $H P(\boldsymbol{v}, c)=\left\{\boldsymbol{x} \in \mathbb{R}_{1}^{n+1} \mid\langle\boldsymbol{x}, \boldsymbol{v}\rangle=c\right\}$. We call $H P(\boldsymbol{v}, c)$ a spacelike hyperplane, a timelike hyperplane or a lightlike hyperplane if $\boldsymbol{v}$ is timelike, spacelike or lightlike respectively.

We now define hyperbolic $n$-space by $H_{+}^{n}(-1)=\left\{\boldsymbol{x} \in \mathbb{R}_{1}^{n+1} \mid\langle\boldsymbol{x}, \boldsymbol{x}\rangle=-1, x_{0} \geq 1\right\}$ and de Sitter $n$-space by $S_{1}^{n}=\left\{\boldsymbol{x} \in \mathbb{R}_{1}^{n+1} \mid\langle\boldsymbol{x}, \boldsymbol{x}\rangle=1\right\}$. Given $\boldsymbol{x}_{1}, \boldsymbol{x}_{2}, \ldots, \boldsymbol{x}_{n} \in \mathbb{R}_{1}^{n+1}$, we define a vector $\boldsymbol{x}_{1} \wedge \boldsymbol{x}_{2} \wedge \cdots \wedge \boldsymbol{x}_{n}$ by

$$
\boldsymbol{x}_{1} \wedge \boldsymbol{x}_{2} \wedge \cdots \wedge \boldsymbol{x}_{n}=\left|\begin{array}{cccc}
-\boldsymbol{e}_{0} & \boldsymbol{e}_{1} & \cdots & \boldsymbol{e}_{n} \\
x_{0}^{1} & x_{1}^{1} & \cdots & x_{n}^{1} \\
x_{0}^{2} & x_{1}^{2} & \cdots & x_{n}^{2} \\
\vdots & \vdots & \cdots & \vdots \\
x_{0}^{n} & x_{1}^{n} & \cdots & x_{n}^{n}
\end{array}\right|,
$$

where $\boldsymbol{e}_{0}, \boldsymbol{e}_{1}, \ldots, \boldsymbol{e}_{n}$ is the canonical basis of $\mathbb{R}_{1}^{n+1}$ and $\boldsymbol{x}_{i}=\left(x_{0}^{i}, x_{1}^{i}, \ldots, x_{n}^{i}\right)$. We can easily show that $\boldsymbol{x}_{1} \wedge \boldsymbol{x}_{2} \wedge \cdots \wedge \boldsymbol{x}_{n}$ is pseudo orthogonal to any $\boldsymbol{x}_{i}(i=1, \ldots, n)$. We also define a set $L C_{+}^{*}=\left\{\boldsymbol{x}=\left(x_{0}, \ldots x_{n}\right) \in L C_{0} \mid x_{0}>0\right\}$, which is called the future lightcone at the origin.

We now construct the local extrinsic differential geometry on hypersurfaces in $H_{+}^{n}(-1)$. Let $\boldsymbol{x}: U \longrightarrow H_{+}^{n}(-1)$ be an embedding, where $U \subset \mathbb{R}^{n-1}$ is an open subset. We shall identify $M=\boldsymbol{x}(U)$ and $U$ through the embedding $\boldsymbol{x}$. Since $\langle\boldsymbol{x}, \boldsymbol{x}\rangle \equiv-1$, we have $\left\langle\boldsymbol{x}_{u_{i}}(u), \boldsymbol{x}(u)\right\rangle \equiv$ $0 \quad(i=1, \ldots, n-1)$, for any $u=\left(u_{1}, \ldots u_{n-1}\right) \in U$. Therefore, if we define

$$
\boldsymbol{e}(u)=\frac{\boldsymbol{x}(u) \wedge \boldsymbol{x}_{u_{1}}(u) \wedge \cdots \wedge \boldsymbol{x}_{u_{n-1}}(u)}{\left\|\boldsymbol{x}(u) \wedge \boldsymbol{x}_{u_{1}}(u) \wedge \cdots \wedge \boldsymbol{x}_{u_{n-1}}(u)\right\|},
$$

we have $\left\langle\boldsymbol{e}(u), \boldsymbol{x}_{u_{i}}(u)\right\rangle \equiv\langle\boldsymbol{e}(u), \boldsymbol{x}(u)\rangle \equiv 0,\langle\boldsymbol{e}(u), \boldsymbol{e}(u)\rangle \equiv 1$, so that $\boldsymbol{x}(u) \pm \boldsymbol{e}(u) \in L C_{+}^{*}$ and hence we can define a map

$$
\mathbb{L}^{ \pm}: U \longrightarrow L C_{+}^{*}
$$


by $\mathbb{L}^{ \pm}(u)=\boldsymbol{x}(u) \pm \boldsymbol{e}(u)$ which is called the hyperbolic Gauss indicatrix (or the lightcone dual) of $\boldsymbol{x}$. In order to define the hyperbolic Gauss-Kronecker curvature of the hypersurface $M=\boldsymbol{x}(U)$, we have shown in [6] that $D_{v} \mathbb{L}^{ \pm} \in T_{p} M$. Here, $D_{v}$ denotes the covariant derivative with respect to the vector $\boldsymbol{v} \in T_{p} M$. This means that $d \mathbb{L}^{ \pm}\left(u_{0}\right)$ is a linear transformation on the tangent space $T_{p} M$. We call the linear transformation $S_{p}^{ \pm}=-d \mathbb{L}^{ \pm}\left(u_{0}\right): T_{p} M \longrightarrow T_{p} M$ the hyperbolic shape operator of $M=\boldsymbol{x}(U)$ at $p=\boldsymbol{x}\left(u_{0}\right)$. We denote the eigenvalues of $S_{p}^{ \pm}$ by $\bar{\kappa}_{p}^{ \pm}$and the eigenvalues of $A_{p}$ by $\kappa_{i}(p)(i=1, \ldots n-1)$ which are called the hyperbolic principal curvatures. The hyperbolic Gauss-Kronecker curvature of $M=\boldsymbol{x}(U)$ at $p=\boldsymbol{x}\left(u_{0}\right)$ is defined to be

$$
K_{h}^{ \pm}\left(u_{0}\right)=\operatorname{det} S_{p}^{ \pm}=\kappa_{1}(p) \cdots \kappa_{n-1}(p) .
$$

We say that a point $p=\boldsymbol{x}(u)$ is an umbilic point if $S_{p}^{ \pm}=\bar{\kappa}^{ \pm}(p) 1_{T_{p} M}$. In [6] the hyperbolic version of the Weingarten formula was shown. Since $\boldsymbol{x}_{u_{i}}(i=1, \ldots n-1)$ are spacelike vectors, we have the Riemannian metric (hyperbolic first fundamental form) given by $d s^{2}=$ $\sum_{i=1}^{n-1} g_{i j} d u_{i} d u_{j}$ on $M=\boldsymbol{x}(U)$, where $g_{i j}(u)=\left\langle\boldsymbol{x}_{u_{i}}(u), \boldsymbol{x}_{u_{j}}(u)\right\rangle$ and the hyperbolic second fundamental invariant defined by $\bar{h}_{i j}^{ \pm}(u)=\left\langle-\mathbb{L}_{u_{i}}^{ \pm}(u), \boldsymbol{x}_{u_{j}}(u)\right\rangle$ for any $u \in U$.

Proposition 2.1. Under the above notations, we have the following formula:

$$
\mathbb{L}_{u_{i}}^{ \pm}=-\sum_{j=1}^{n-1}\left(\bar{h}^{ \pm}\right)_{i}^{j} \boldsymbol{x}_{u_{j}} \text { (The hyperbolic Weingarten formula), }
$$

where $\left(\left(\bar{h}^{ \pm}\right)_{i}^{j}\right)=\left(\bar{h}_{i k}^{ \pm}\right)\left(g^{k j}\right)$ and $\left(g^{k j}\right)=\left(g_{k j}\right)^{-1}$. Therefore we have the following formula:

$$
K_{h}^{ \pm}=\frac{\operatorname{det}\left(\bar{h}_{i j}^{ \pm}\right)}{\operatorname{det}\left(g_{\alpha \beta}\right)} .
$$

In the previous paragraphs we reviewed the properties of hyperbolic Gauss indicatrices and hyperbolic Gauss-Kronecker curvatures. The original definition of the hyperbolic Gauss map introduced by Bryant [1] and Epstein [5] is given in the Poincaré ball model. Here, we introduce the corresponding definition in Minkowski model as follows: If $\boldsymbol{x}=\left(x_{0}, x_{1}, \ldots, x_{n}\right)$ is a lightlike vector, then $x_{0} \neq 0$. Therefore we have

$$
\tilde{\boldsymbol{x}}=\left(1, \frac{x_{1}}{x_{0}}, \ldots, \frac{x_{n}}{x_{0}}\right) \in S_{+}^{n-1}=\left\{\boldsymbol{x}=\left(x_{0}, x_{1}, \ldots, x_{n}\right) \mid\langle\boldsymbol{x}, \boldsymbol{x}\rangle=0, x_{0}=1\right\} .
$$

We call $S_{+}^{n-1}$ the lightcone $(n-1)$-sphere. We define a map

$$
\widetilde{\mathbb{L}}^{ \pm}: U \longrightarrow S_{+}^{n-1}
$$

by $\widetilde{\mathbb{L}}^{ \pm}(u)=\widetilde{\mathbb{L}^{ \pm}(u)}$ and call it the hyperbolic Gauss map of $\boldsymbol{x}$. We remark that for $n=3$, our definition of hyperbolic Gauss map is equivalent to the one introduced in $[1,5]$. Let $N_{p} M$ be the pseudo-normal space of $T_{p} M$ in $T_{p} \mathbb{R}_{1}^{n+1}$. We have the decomposition $T_{p} \mathbb{R}_{1}^{n+1}=$ $T_{p} M \oplus N_{p} M$, so that we also have the Whitney sum $T \mathbb{R}^{n+1}=T M \oplus N M$. Therefore we have the canonical projection $\Pi: T \mathbb{R}^{n+1} \longrightarrow T M$. It follows that we have a linear transformation $\Pi_{p} \circ d \widetilde{\mathbb{L}}^{ \pm}(u): T_{p} M \longrightarrow T_{p} M$ for $p=\boldsymbol{x}(u)$ by the identification of $U$ and $\boldsymbol{x}(U)=M$ via $\boldsymbol{x}$. In $[12]$ the following formula was shown: 
Proposition 2.2. Under the above notation we have the following horospherical Weingarten formula:

$$
\Pi_{p} \circ \widetilde{\mathbb{L}}_{u_{i}}^{ \pm}=-\sum_{j=1}^{n-1} \frac{1}{\ell_{0}^{ \pm}(u)}\left(\bar{h}^{ \pm}\right)_{i}^{j} \boldsymbol{x}_{u_{j}},
$$

where $\mathbb{L}^{ \pm}(u)=\left(\ell_{0}^{ \pm}(u), \ell_{1}^{ \pm}(u), \ldots, \ell_{n}^{ \pm}(u)\right)$.

We call the linear transformation $\widetilde{S}_{p}^{ \pm}=-\Pi_{p} \circ d \widetilde{\mathbb{L}}^{ \pm}$the horospherical shape operator of $M=\boldsymbol{x}(U)$. We also define the principal horospherical curvature $\widetilde{\kappa}_{i}^{ \pm}(p)(i=1, \ldots, n-1)$ as the eigenvalues of $\widetilde{S}_{p}^{ \pm}$. By the above proposition, we have $\widetilde{\kappa}_{i}^{ \pm}(p)=\left(1 / \ell_{0}^{ \pm}\right) \bar{\kappa}_{i}^{ \pm}(p)$. The horospherical Gauss-Kronecker curvature of $\boldsymbol{x}(U)=M$ is defined to be $\widetilde{K}_{h}^{ \pm}(u)=\operatorname{det} \widetilde{S}_{p}^{ \pm}$. It follows that we have the following relation between the horospherical Gauss-Kronecker curvature and the hyperbolic Gauss-Kronecker curvature:

$$
\widetilde{K}_{h}^{ \pm}(u)=\left(\frac{1}{\ell_{0}^{ \pm}(u)}\right)^{n-1} K_{h}^{ \pm}(u) .
$$

We remark that $\widetilde{\kappa}_{i}^{ \pm}(p)$ is not invariant under hyperbolic motions but it is an $S O(n)$-invariant. We also remark that the notion of horospherical curvatures is independent of the choice of the model of hyperbolic space. For the purpose, we introduce a smooth function on the unit tangent sphere bundle of hyperbolic space which plays the principal role of the horospherical geometry. Let $S O_{0}(n, 1)$ be the identity component of the matrix group

$$
S O(n, 1)=\left\{g \in G L(n+1, \mathbb{R}) \mid g I_{n, 1}{ }^{t} g=I_{n, 1}, \quad\right\},
$$

where

$$
I_{n, 1}=\left(\begin{array}{c|c}
-1 & \mathbf{0} \\
\hline{ }^{t} \mathbf{0} & I_{n}
\end{array}\right) \in G L(n+1, \mathbb{R}) .
$$

It is well-known that $S O_{0}(n, 1)$ acts transitively on $H_{+}^{n}(-1)$ and the isotropic group at $p=$ $(1,0, \ldots, 0)$ is $S O(n)$ which is naturally embedded in $S O_{0}(n, 1)$. Moreover the action induces isometries on $H_{+}^{n}(-1)$. On the other hand, we consider a submanifold $\Delta=\{(\boldsymbol{v}, \boldsymbol{w}) \mid\langle\boldsymbol{v}, \boldsymbol{w}\rangle=$ $0\}$ of $H_{+}^{n}(-1) \times S_{1}^{n}$ and the canonical projection $\bar{\pi}: \Delta \longrightarrow H_{+}^{n}(-1)$. Let $\pi: S\left(T H_{+}^{n}(-1)\right) \longrightarrow$ $H^{n}(-1)$ be the unit tangent sphere bundle over $H_{+}^{n}(-1)$. For any $\boldsymbol{v} \in H_{+}^{n}(-1)$, we have the local (global) coordinates $\left(v_{1}, \ldots, v_{n}\right)$ of $H_{+}^{n}(-1)$ such that $\boldsymbol{v}=\left(\sqrt{v_{1}^{2}+\cdots+v_{n}^{2}+1}, v_{1}, \ldots, v_{n}\right)$. We can represent the tangent vector $\boldsymbol{w}=\sum_{i=1}^{n} w_{i} \partial / \partial v_{i} \in T_{v} H_{+}^{n}(-1)$ by

$$
\boldsymbol{w}=\left(\frac{1}{v_{0}} \sum_{i=1}^{n} w_{i} v_{i}, w_{1}, \ldots, w_{n}\right)
$$

as a vector in Minkowski $(n+1)$-space. Then $\langle\boldsymbol{w}, \boldsymbol{v}\rangle=\left(-\frac{1}{v_{0}} \sum_{i=1}^{n} w_{i} v_{i}\right) v_{0}+\sum_{i=1}^{n} w_{i} v_{i}=0$. Therefore $\boldsymbol{w} \in S\left(T_{v} H_{+}^{n}(-1)\right)$ if and only if

$$
\langle\boldsymbol{w}, \boldsymbol{w}\rangle=1 \text { and }\langle\boldsymbol{v}, \boldsymbol{w}\rangle=0 .
$$

The above conditions are equivalent to the condition $(\boldsymbol{v}, \boldsymbol{w}) \in \Delta$. This means that we can canonically identify $\pi: S\left(T H_{+}^{n}(-1)\right) \longrightarrow H_{+}^{n}(-1)$ with $\bar{\pi}: \Delta \longrightarrow H_{+}^{n}(-1)$. Moreover, the 
linear action of $S O_{0}(n, 1)$ on $\mathbb{R}_{1}^{n+1}$ induces the canonical action on $\Delta$ (i.e., $g(\boldsymbol{v}, \boldsymbol{w})=(g \boldsymbol{v}, g \boldsymbol{w})$ for any $\left.g \in S O_{0}(n, 1)\right)$. For any $(\boldsymbol{v}, \boldsymbol{w}) \in \Delta$, the first component of $\boldsymbol{v} \pm \boldsymbol{w}$ is given by

$$
v_{0} \pm w_{0}=\sqrt{v_{1}^{2}+\cdots+v_{n}^{2}+1} \pm \frac{1}{\sqrt{v_{1}^{2}+\cdots+v_{n}^{2}+1}} \sum_{i=1}^{n} v_{i} w_{i}
$$

so that it can be considered as a function on the unit tangent bundle $S\left(T H_{+}^{n}(-1)\right)$.

We now define a function

$$
\mathcal{N}_{h}^{ \pm}: \Delta \longrightarrow \mathbb{R} ; \mathcal{N}_{h}^{ \pm}(\boldsymbol{v}, \boldsymbol{w})=\frac{1}{v_{0} \pm w_{0}} .
$$

We call $\mathcal{N}_{h}^{ \pm}$a horospherical normalization function on $H_{+}^{n}(-1)$. Since $v^{2}+\cdots+v_{n}^{2}+1$ and $\sum_{i=1}^{n} v_{i} w_{i}$ are $S O(n)$-invariant functions, $\mathcal{N}_{h}^{ \pm}$is an $S O(n)$-invariant function. Therefore, $\mathcal{N}_{h}^{ \pm}$ can be considered as a function on the unit tangent sphere bundle over the hyperbolic space $S O_{0}(n, 1) / S O(n)$ which is independent of the choice of the model space. For any embedding $\boldsymbol{x}: U \longrightarrow H_{+}^{n}(-1)\left(U \subset \mathbb{R}^{n-1}\right)$, we have the unit normal vector field $\boldsymbol{e}: U \longrightarrow S_{1}^{n}$, so that $(\boldsymbol{x}(u), \boldsymbol{e}(u)) \in \Delta$ for any $u \in U$. It follows that

$$
\widetilde{K}_{h}^{ \pm}(u)=\mathcal{N}_{h}^{ \pm}(\boldsymbol{x}(u), \boldsymbol{e}(u))^{n-1} K_{h}^{ \pm}(u) .
$$

The right hand side of the above equality is independent of the choice of the model space.

We now consider the global properties of curvatures. Let $M$ be a closed orientable $(n-1)$ dimensional manifold and $f: M \longrightarrow H_{+}^{n}(-1)$ an embedding. We consider the canonical projection $\pi: \mathbb{R}_{1}^{n+1} \longrightarrow \mathbb{R}^{n}$ defined by $\pi\left(x_{0}, x_{1}, \ldots, x_{n}\right)=\left(0, x_{1}, \ldots, x_{n}\right)$. Then we have orientation preserving diffeomorphisms $\pi \mid H_{+}^{n}(-1): H_{+}^{n}(-1) \longrightarrow \mathbb{R}^{n}$ and $\pi \mid S_{+}^{n-1}: S_{+}^{n-1} \longrightarrow$ $S^{n-1}$. Consider the outward unit normal $\mathbb{E}$ of $f(M)$ in $H_{+}^{n}(-1)$, then we define the hyperbolic Gauss indicatrix in the global

by

$$
\mathbb{L}: M \longrightarrow L C_{+}^{*}
$$

$$
\mathbb{L}(p)=f(p)+\mathbb{E}(p) .
$$

The global hyperbolic Gauss-Kronecker curvature function $\mathcal{K}_{h}: M \longrightarrow \mathbb{R}$ is then defined in the usual way in terms of the global hyperbolic Gauss indicatrix $\mathbb{L}$. We also define the hyperbolic Gauss map in the global

by

$$
\widetilde{\mathbb{L}}: M \longrightarrow S_{+}^{n-1}
$$

$$
\widetilde{\mathbb{L}}(p)=\widetilde{\mathbb{L}(p)} .
$$

We now define a global horospherical Gauss-Kronecker curvature function $\widetilde{\mathcal{K}}_{h}: M \longrightarrow \mathbb{R}$ by

$$
\widetilde{\mathcal{K}}_{h}(p)=\mathcal{N}_{h}(f(p), \mathbb{E}(p))^{n-1} \mathcal{K}_{h}(p),
$$

where we simply write $\mathcal{N}_{h}=\mathcal{N}_{h}^{+}$. In [12] the following Gauss-Bonnet type theorem for the horospherical Gauss-Kronecker curvature was shown.

Theorem 2.3. If $M$ is a closed orientable even-dimensional hypersurface in hyperbolic $n$ space, then

$$
\int_{M} \widetilde{\mathcal{K}}_{h} d \mathfrak{v}_{M}=\frac{1}{2} \gamma_{n-1} \chi(M)
$$


where $\chi(M)$ is the Euler characteristic of $M, d \mathfrak{v}_{M}$ is the volume form of $M$ and the constant $\gamma_{n-1}$ is the volume of the unit $(n-1)$-sphere $S^{n-1}$.

In [11] the horospherical geometry on general submanifolds in $H_{+}^{n}(-1)$ was studied. Let $\boldsymbol{x}: U \rightarrow H_{+}^{n}(-1)$ be an embedding of codimension $(r+1)$, where $U \subset \mathbb{R}^{s}$ is an open subset $(s+r+1=n)$. We also write that $M=\boldsymbol{x}(U)$ and identify $M$ and $U$ through the embedding $\boldsymbol{x}$. For any $p=\boldsymbol{x}(u) \in M \subset H_{+}^{n}(-1)$, we have $\langle\boldsymbol{x}(u), \boldsymbol{x}(u)\rangle=-1$, so that $\left\langle\boldsymbol{x}_{u_{i}}(u), \boldsymbol{x}(u)\right\rangle=0$, where $u=\left(u_{1}, u_{2}, \ldots, u_{s}\right)$. The tangent space of $M$ at $p=\boldsymbol{x}(u)$ is

$$
T_{p} M=\left\langle\boldsymbol{x}_{u_{1}}(u), \boldsymbol{x}_{u_{2}}(u), \ldots, \boldsymbol{x}_{u_{s}}(u)\right\rangle_{\mathbb{R}}
$$

Let $N_{p}(M)$ be the normal space of $M$ at $p=\boldsymbol{x}(u)$ in $\mathbb{R}_{1}^{n+1}$ and we define $N_{p}^{h}(M)=N_{p}(M) \cap$ $T_{p} H_{+}^{n}(-1)$. Since the normal bundle $N(M)$ is trivial, we can arbitrarily choose a unit normal section $\boldsymbol{n}(u) \in S^{r}\left(N_{p}^{h}(M)\right)$. We can consider the differential geometry of general submanifolds in hyperbolic space which generalizes the differential geometry of hypersurfaces in hyperbolic space in [6]. Since $\langle\boldsymbol{n}(u), \boldsymbol{n}(u)\rangle=1$ and $\langle\boldsymbol{x}(u), \boldsymbol{n}(u)\rangle=0, \boldsymbol{n}_{u_{i}}(u)(i=1, \ldots, s)$ are orthogonal to both of $\boldsymbol{n}(u)$ and $\boldsymbol{x}(u)$. Therefore we have $\boldsymbol{n}_{u_{i}}(u) \in T_{p} M \oplus N_{p}^{h}(M)$.

We now consider the orthogonal projections $\pi^{T}: T_{p} M \oplus N_{p}^{h}(M) \longrightarrow T_{p} M$ and $\pi^{N}: T_{p} M \oplus$ $N_{p}^{h}(M) \longrightarrow N_{p}^{h}(M)$. Let $d \boldsymbol{n}_{u}: T_{u} U \longrightarrow T_{p} M \oplus N_{p}^{h}(M)$ be the derivative of $\boldsymbol{n}$. We define that $d \boldsymbol{n}_{u}^{T}=\pi^{T} \circ d \boldsymbol{n}_{u}$ and $d \boldsymbol{n}_{u}^{N}=\pi^{N} \circ d \boldsymbol{n}_{u}$. We call the linear transformation $A_{p_{0}}(\boldsymbol{n})=-d \boldsymbol{n}_{u_{0}}^{T}$ : $T_{p_{0}} M \longrightarrow T_{p_{0}} M$ the $\boldsymbol{n}$-shape operator of $M=\boldsymbol{x}(U)$ at $p_{0}=\boldsymbol{x}\left(u_{0}\right)$. Under the identification of $U$ and $M$, the derivative $d \boldsymbol{x}_{u}$ can be identified with the identity mapping $\operatorname{id}_{T_{p} M}$. We also call the linear transformation $S_{p_{0}}(\boldsymbol{n})=-\left(\mathrm{id}_{T_{p_{0}} M}+d \boldsymbol{n}_{u_{0}}^{T}\right): T_{p_{0}} M \longrightarrow T_{p_{0}} M$ the horospherical (or, hyperbolic ) $\boldsymbol{n}$-shape operator of $M=\boldsymbol{x}(U)$ at $p_{0}=\boldsymbol{x}\left(u_{0}\right)$. We also call the linear mapping $d \boldsymbol{n}_{u}^{N}$ the normal connection with respect to $\boldsymbol{n}$ of $M=\boldsymbol{x}(U)$ at $p_{0}=\boldsymbol{x}\left(u_{0}\right)$. We denote the eigenvalue of $A_{p_{0}}(\boldsymbol{n})$ by $\kappa_{p_{0}}(\boldsymbol{n})$ and the eigenvalue of $S_{p_{0}}(\boldsymbol{n})$ by $\bar{\kappa}_{p_{0}}(\boldsymbol{n})$. By the relation of $A_{p_{0}}(\boldsymbol{n})$ and $S_{p_{0}}(\boldsymbol{n})$ we have a relation $\bar{\kappa}_{p_{0}}(\boldsymbol{n})=\kappa_{p_{0}}(\boldsymbol{n})-1$. We call $\bar{\kappa}_{p_{0}}(\boldsymbol{n})$ the horospherical principal curvature at $p_{0}$ with respect to $\boldsymbol{n}$.

We now define the notion of curvature as follows. The horospherical (or hyperbolic) curvature with respect to $\boldsymbol{n}$ at $p_{0}=\boldsymbol{x}\left(u_{0}\right)$ is defined to be

$$
K_{h}(\boldsymbol{n})\left(\boldsymbol{x}\left(u_{0}\right)\right)=K_{h}(\boldsymbol{n})_{p_{0}}=\operatorname{det} S_{p_{0}}(\boldsymbol{n}) .
$$

We give the following generalized hyperbolic Weingarten formula. Since $\boldsymbol{x}_{u_{i}}(i=1, \ldots s)$ are spacelike vectors, we induce the Riemannian metric (the hyperbolic first fundamental form ) $d s^{2}=\sum_{i=1}^{s} g_{i j} d u_{i} d u_{j}$ on $M=\boldsymbol{x}(U)$, where $g_{i j}(u)=\left\langle\boldsymbol{x}_{u_{i}}(u), \boldsymbol{x}_{u_{j}}(u)\right\rangle$ for any $u \in U$. We also define the horospherical (or, hyperbolic) second fundamental invariant with respect to the unit normal vector field $\boldsymbol{n}$ by $\bar{h}_{i j}(\boldsymbol{n})(u)=\left\langle-(\boldsymbol{x}+\boldsymbol{n})_{u_{i}}(u), \boldsymbol{x}_{u_{j}}(u)\right\rangle$ for any $u \in U$. If we define the second fundamental invariant with respect to the normal vector field $\boldsymbol{n}$ by $h_{i j}(\boldsymbol{n})(u)=$ $-\left\langle\boldsymbol{n}_{u_{i}}(u), \boldsymbol{x}_{u_{j}}(u)\right\rangle$, then we have the following relation:

$$
\bar{h}_{i j}(\boldsymbol{n})(u)=-g_{i j}(u)+h_{i j}(\boldsymbol{n})(u),(i, j=1, \ldots, s) .
$$

Proposition 2.4. Under the above notations, we have the following horospherical (or, hyperbolic) Weingarten formula with respect to $\boldsymbol{n}$ :

$$
\pi^{T} \circ(\boldsymbol{x}+\boldsymbol{n})_{u_{i}}=-\sum_{j=1}^{s} \bar{h}_{i}^{j}(\boldsymbol{n}) \boldsymbol{x}_{u_{j}},
$$


where $\left(\bar{h}_{i}^{j}(\boldsymbol{n})\right)=\left(\bar{h}_{i k}(\boldsymbol{n})\right)\left(g^{k j}\right)$ and $\left(g^{k j}\right)=\left(g_{k j}\right)^{-1}$. It follows that the horospherical curvature with respect to $\boldsymbol{n}$ is given by

$$
K_{h}(\boldsymbol{n})(\boldsymbol{x}(u))=\frac{\operatorname{det}\left(\bar{h}_{i j}(\boldsymbol{n})(u)\right)}{\operatorname{det}\left(g_{\alpha \beta}(u)\right)} .
$$

Since $\left\langle-(\boldsymbol{x}+\boldsymbol{n})(u), \boldsymbol{x}_{u_{j}}(u)\right\rangle=0$, we have $\bar{h}_{i j}(\boldsymbol{n})(u)=\left\langle\boldsymbol{x}(u)+\boldsymbol{n}(u), \boldsymbol{x}_{u_{i} u_{j}}(u)\right\rangle$. Therefore the horospherical second fundamental invariant at a point $p_{0}=\boldsymbol{x}\left(u_{0}\right)$ depends only on $\boldsymbol{x}\left(u_{0}\right)+$ $\boldsymbol{n}\left(u_{0}\right)$ and $\boldsymbol{x}_{u_{i} u_{j}}\left(u_{0}\right)$. By the above corollary, the horospherical curvature also depends only on $\boldsymbol{x}\left(u_{0}\right)+\boldsymbol{n}\left(u_{0}\right)$ and $\boldsymbol{x}_{u_{i} u_{j}}\left(u_{0}\right)$. It is independent on the choice of the normal vector field $\boldsymbol{n}$. We write $K_{h}\left(\boldsymbol{n}_{0}\right)\left(u_{0}\right)$ as the horospherical curvature at $p_{0}=\boldsymbol{x}\left(u_{0}\right)$ with respect to $\boldsymbol{n}_{0}=\boldsymbol{n}\left(u_{0}\right)$. We might also say that a point $p_{0}=\boldsymbol{x}\left(u_{0}\right)$ is $\boldsymbol{n}_{0}$-umbilic because the horospherical $\boldsymbol{n}$-shape operator is independent on the choice of the normal vector field $\boldsymbol{n}$ (it depends on the normal vector $\left.\boldsymbol{n}_{0}=\boldsymbol{n}\left(u_{0}\right)\right)$.

We now arbitrarily choose unit orthonormal sections $\boldsymbol{n}_{j}(u) \in S^{r}\left(N_{p}^{h}(M)\right)(j=1, \ldots, r+1)$. Therefore we have

$$
N_{p}(M)=\left\langle\boldsymbol{x}(u), \boldsymbol{n}_{1}(u), \ldots, \boldsymbol{n}_{r+1}(u)\right\rangle_{\mathbb{R}}
$$

Since $\left\{\boldsymbol{x}, \boldsymbol{x}_{u_{1}}, \ldots, \boldsymbol{x}_{u_{s}}, \boldsymbol{n}_{1}, \ldots, \boldsymbol{n}_{r+1}\right\}$ is a frame of $T \mathbb{R}_{1}^{n+1}$ along $M$, we have $\left(\boldsymbol{n}_{j}\right)_{u_{i}}=\sum_{i=1}^{s} \lambda_{i} \boldsymbol{x}_{u_{i}}+$ $\sum_{k=1}^{r+1} \mu_{k} \boldsymbol{n}_{k}+\mu_{r+2} \boldsymbol{x}$ for some $\lambda_{i}, \mu_{k} \in \mathbb{R}, i=1,2, \ldots, s, j=1, \ldots, r+2$, where we denote $\left(\boldsymbol{n}_{j}\right)_{u_{i}}=\left(\partial \boldsymbol{n}_{j} / \partial u_{i}\right)(u)$. It follows from the fact $\left\langle\boldsymbol{n}_{j}, \boldsymbol{n}_{j}\right\rangle=1$ that $\left\langle\left(\boldsymbol{n}_{j}\right)_{u_{i}}, \boldsymbol{n}_{j}\right\rangle=0$. Thus we have $\mu_{j}=0$. By the relation $\left\langle\boldsymbol{x}, \boldsymbol{n}_{j}\right\rangle=0$, we have $\left\langle\boldsymbol{x},\left(\boldsymbol{n}_{j}\right)_{u_{i}}\right\rangle=-\left\langle\boldsymbol{x}_{u_{i}}, \boldsymbol{n}_{j}\right\rangle=0$. Hence $\mu_{r+2}=0$. Therefore, we have a relation

$$
\left(\boldsymbol{n}_{j}\right)_{u_{i}}(u) \in\left\langle\boldsymbol{x}_{u_{1}}(u), \ldots, \boldsymbol{x}_{u_{s}}(u), \boldsymbol{n}_{1}(u) \ldots, \boldsymbol{n}_{j-1}(u), \boldsymbol{n}_{j+1}(u), \ldots, \boldsymbol{n}_{r+1}(u)\right\rangle_{\mathbb{R}},
$$

\section{Total Absolute Horospherical Curvature}

In this section $M$ denotes a compact $s$-dimensional manifold and $f: M^{s} \rightarrow H_{+}^{n}(-1)$ denotes an immersion of codimension $(r+1)$. Let $\nu^{1} M$ denote the unitary normal bundle of the immersion $f$, i.e.:

$$
\nu^{1} M=\left\{(p, \xi) ; \xi \in N_{p}^{h}(M) \text { and }\langle\xi, \xi\rangle=1\right\} .
$$

Definition 3.1. The horospherical Gauss map $\widetilde{\mathbb{L}}: \nu^{1} M \rightarrow S_{+}^{n-1}$ of the immersion $f: M^{s} \rightarrow$ $H_{+}^{n}(-1)$ is defined by the following commutative diagram

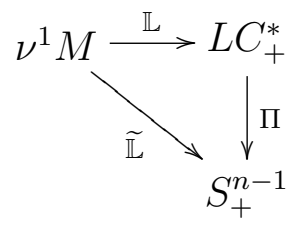

where $\mathbb{L}: \nu^{1} M \rightarrow L C_{+}^{*} ; \mathbb{L}(p, \xi)=f(p)+\xi$ is the hyperbolic Gauss indicatrix of the immersion $f$ and $\Pi(\boldsymbol{v})=\widetilde{\boldsymbol{v}}$.

The horospherical Gauss map lead us to a curvature in the framework of horospherical geometry. Let $T_{(\boldsymbol{x}, \boldsymbol{n})} \nu^{1} M$ be the tangent space of $\nu^{1} M$ at $(\boldsymbol{x}, \boldsymbol{n})$. We have the canonical identification $T_{(\boldsymbol{x}, \boldsymbol{n})} \nu^{1} M=T_{\boldsymbol{x}} M \oplus T_{\boldsymbol{n}} S^{r} \subset T_{\boldsymbol{x}} M \oplus N_{\boldsymbol{x}} M=T_{\boldsymbol{x}} \mathbb{R}_{1}^{n+1}$, where $N_{\boldsymbol{x}} M$ is the normal 
vector space of $M$ at $\boldsymbol{x}$ in $\mathbb{R}_{1}^{n+1}$. Let $P: \widetilde{\mathbb{L}}^{*} T \mathbb{R}_{1}^{n+1}=T \nu^{1} M \oplus \mathbb{R}^{2} \rightarrow T \nu^{1} M$ be the canonical projection. It follows that we have a linear transformation

$$
P_{\widetilde{\mathbb{L}}(\boldsymbol{x}, \boldsymbol{n})} \circ d \widetilde{\mathbb{L}}: T_{(\boldsymbol{x}, \boldsymbol{n})} \nu^{1} M \rightarrow T_{(\boldsymbol{x}, \boldsymbol{n})} \nu^{1} M .
$$

Definition 3.2. The horospherical curvature with respect to $\boldsymbol{n}$ at $\boldsymbol{x}$ is defined to be

$$
\widetilde{K}_{h}(\boldsymbol{x}, \boldsymbol{n})=\operatorname{det}\left(P_{\widetilde{\mathbb{L}}(\boldsymbol{x}, \boldsymbol{n})} \circ(-d \widetilde{\mathbb{L}})\right) .
$$

Let $\boldsymbol{x}:(U, 0) \rightarrow\left(H_{+}^{n}(-1), \boldsymbol{x}(0)\right)$ be an embedding and let $\left\{\boldsymbol{n}_{1}(u), \ldots, \boldsymbol{n}_{r}(u), \boldsymbol{n}_{r+1}(u)\right\}$ be orthonormal normal vector fields on $\boldsymbol{x}(U)$ such that $\boldsymbol{n}_{r+1}=\boldsymbol{n}$. Since $\left\{\boldsymbol{x}_{u_{1}}, \ldots, \boldsymbol{x}_{u_{s}}, \boldsymbol{n}_{1}, \ldots, \boldsymbol{n}_{r}\right\}$ is a basis of $T_{(\boldsymbol{x}, \boldsymbol{n})} \nu^{1} M$ and writing $\left(\ell_{0}, \ell_{1}, \ldots, \ell_{n+1}\right)$ as the coordinates of $\mathbb{L}$,

$$
\begin{aligned}
K_{h}(\boldsymbol{n})(\boldsymbol{x}(u)) & =\operatorname{det}\left(\left(\left\langle\pi^{T} \circ(\boldsymbol{x}+\boldsymbol{n})_{u_{i}}, \boldsymbol{x}_{u_{j}}\right\rangle\right)\left(g^{i j}\right)\right) \\
& =\operatorname{det}\left(\left(\left\langle(\boldsymbol{x}+\boldsymbol{n})_{u_{i}}, \boldsymbol{x}_{u_{j}}\right\rangle\right)\left(g^{i j}\right)\right) \\
& =\operatorname{det}\left(\left(\begin{array}{cc}
\left\langle(\boldsymbol{x}+\boldsymbol{n})_{u_{i}}, \boldsymbol{x}_{u_{j}}\right\rangle & \left\langle(\boldsymbol{x}+\boldsymbol{n})_{u_{i}}, \boldsymbol{n}_{j}\right\rangle \\
1 \leqslant i, j \leqslant s & 1 \leqslant i \leqslant s ; 1 \leqslant j \leqslant r \\
\mathbf{0}_{r \times s} & I_{r}
\end{array}\right)\left(\begin{array}{cc}
g^{i j} & \mathbf{0} \\
\mathbf{0} & I_{r}
\end{array}\right)\right)
\end{aligned}
$$

Since $\ell_{0} \widetilde{\mathbb{L}}=\mathbb{L}$, we have $\left(\ell_{0}\right) \mathbb{L}_{u_{i}}=\mathbb{L}_{u_{i}}-\left(\ell_{0}\right)_{u_{i}} \widetilde{\mathbb{L}}$. It follows that $P_{\widetilde{L}}\left(\left(\ell_{0}\right) \mathbb{L}_{u_{i}}\right)=\mathbb{L}_{u_{i}}$, so that we have

$$
\begin{aligned}
\widetilde{K}_{h}(\boldsymbol{x}, \boldsymbol{n})(u) & =\operatorname{det}\left(\begin{array}{cc}
\frac{1}{\ell_{0}}\left\langle(\boldsymbol{x}+\boldsymbol{n})_{u_{i}}, \boldsymbol{x}_{u_{j}}\right\rangle & \frac{1}{\ell_{0}}\left\langle(\boldsymbol{x}+\boldsymbol{n})_{u_{i}}, \boldsymbol{n}_{j}\right\rangle \\
1 \leqslant i, j \leqslant s & 1 \leqslant i \leqslant s ; 1 \leqslant j \leqslant r \\
\mathbf{0}_{r \times s} & \frac{1}{\ell_{0}} I_{r}
\end{array}\right) \\
& =\frac{1}{\ell_{0}^{(s+r)}} \operatorname{det}\left(\left\langle(\boldsymbol{x}+\boldsymbol{n})_{u_{i}}, \boldsymbol{x}_{u_{j}}\right\rangle\right) \\
& =\frac{1}{\ell_{0}^{(s+r)}} \operatorname{det}\left(\left\langle\pi^{T} \circ(\boldsymbol{x}+\boldsymbol{n})_{u_{i}}, \boldsymbol{x}_{u_{j}}\right\rangle\right) \\
& =\frac{1}{\ell_{0}^{(s+r)}} K_{h}(\boldsymbol{n})(\boldsymbol{x}(u))=\mathcal{N}_{h}^{s+r}(\boldsymbol{x}, \boldsymbol{n}) K_{h}(\boldsymbol{n})(\boldsymbol{x}(u)) .
\end{aligned}
$$

Lemma 3.3. If $\bar{\delta}$ denotes the canonical volume density of $\nu^{1} M$ and $\sigma$ denotes the canonical volume density of the lightcone sphere $S_{+}^{n-1}$ then

$$
\left(\widetilde{\mathbb{L}}^{*} \sigma\right)_{(\boldsymbol{x}, \boldsymbol{n})}=\left|\widetilde{K}_{h}(\boldsymbol{x}, \boldsymbol{n})\right| \bar{\delta}_{(\boldsymbol{x}, \boldsymbol{n})},
$$

for all $(\boldsymbol{x}, \boldsymbol{n}) \in \nu^{1} M$.

Proof. Under the above notation it suffices to show that if $(\boldsymbol{x}, \boldsymbol{n})$ is a nonsingular point of $\widetilde{\mathbb{L}}$ then $\operatorname{det}\left(\left\langle\partial_{i} \widetilde{\mathbb{L}}, \partial_{j} \widetilde{\mathbb{L}}\right\rangle\right)=\left(\widetilde{K}_{h}(\boldsymbol{x}, \boldsymbol{n})\right)^{2} \operatorname{det}\left(\left\langle\partial_{i} \boldsymbol{x}, \partial_{j} \boldsymbol{x}\right\rangle\right)$. Locally we may write

$$
\mathbb{L}(u, t)=\boldsymbol{x}(u)+t_{1} \boldsymbol{n}_{1}(u)+\cdots+t_{r} \boldsymbol{n}_{r}(u)+\sqrt{1-\sum_{i=1}^{r} t_{i}^{2} \boldsymbol{n}(u)} .
$$


TOTAL ABSOLUTE HOROSPHERICAL CURVATURE OF SUBMANIFOLDS IN HYPERBOLIC SPACE 9 Since $\ell_{0} \widetilde{\mathbb{L}}=\mathbb{L}$, we calculate that $\left(\left(\ell_{0}\right) \partial_{i} \widetilde{\mathbb{L}}\right)_{(0,0)}=\left(\partial_{i} \mathbb{L}-\left(\partial_{i} \ell_{0}\right) \widetilde{\mathbb{L}}\right)_{(0,0)}$, where $\mathbb{L}=\left(\ell_{0}\right.$, $\left.\ell_{1}, \ldots, \ell_{n+1}\right)$. It follows that

$$
\begin{aligned}
& \widetilde{g}=\left(\begin{array}{cc}
\left\langle\widetilde{\mathbb{L}}_{u_{i}}, \widetilde{\mathbb{L}}_{u_{j}}\right\rangle & \left\langle\widetilde{\mathbb{L}}_{u_{i}}, \widetilde{\mathbb{L}}_{t_{j}}\right\rangle \\
1 \leqslant i, j \leqslant s & 1 \leqslant i \leqslant s ; 1 \leqslant j \leqslant r \\
\left\langle\widetilde{\mathbb{L}}_{u_{j}}, \widetilde{\mathbb{L}}_{t_{i}}\right\rangle & \left\langle\widetilde{\mathbb{L}}_{t_{i}}, \widetilde{\mathbb{L}}_{t_{j}}\right\rangle \\
1 \leqslant j \leqslant s ; 1 \leqslant i \leqslant r & 1 \leqslant i, j \leqslant r
\end{array}\right) \\
& =\left(\frac{1}{\ell_{0}}\right)^{2}\left(\begin{array}{cc}
\left\langle(\boldsymbol{x}+\boldsymbol{n})_{u_{i}},(\boldsymbol{x}+\boldsymbol{n})_{u_{j}}\right\rangle & \left\langle(\boldsymbol{x}+\boldsymbol{n})_{u_{i}}, \boldsymbol{n}_{j}\right\rangle \\
1 \leqslant i, j \leqslant s & 1 \leqslant i \leqslant s ; 1 \leqslant j \leqslant r \\
\left\langle(\boldsymbol{x}+\boldsymbol{n})_{u_{j}}, \boldsymbol{n}_{i}\right\rangle & \left\langle\boldsymbol{n}_{i}, \boldsymbol{n}_{j}\right\rangle \\
1 \leqslant j \leqslant s ; 1 \leqslant i \leqslant r & 1 \leqslant i, j \leqslant r
\end{array}\right) \\
& =\left(\frac{1}{\ell_{0}}\right)^{2}\left(\begin{array}{cc}
\left(\left\langle\pi^{T} \circ(\boldsymbol{x}+\boldsymbol{n})_{u_{i}}, \pi^{T} \circ(\boldsymbol{x}+\boldsymbol{n})_{u_{j}}\right\rangle+\sum_{l}\left\langle\boldsymbol{n}_{u_{i}}, \boldsymbol{n}_{l}\right\rangle\left\langle\boldsymbol{n}_{u_{j}}, \boldsymbol{n}_{l}\right\rangle\right) & \left\langle\boldsymbol{n}_{u_{i}}, \boldsymbol{n}_{j}\right\rangle \\
1 \leqslant i, j \leqslant s & 1 \leqslant i \leqslant s ; 1 \leqslant j \leqslant r \\
\left\langle\boldsymbol{n}_{u_{j}}, \boldsymbol{n}_{i}\right\rangle & I_{r} \\
1 \leqslant j \leqslant s ; 1 \leqslant i \leqslant r &
\end{array}\right) . \\
& \widetilde{g}_{0}=\left(\frac{1}{\ell_{0}}\right)^{2}\left(\begin{array}{cc}
\left\langle\pi^{T} \circ(\boldsymbol{x}+\boldsymbol{n})_{u_{i}, \pi^{T}} \circ(\boldsymbol{x}+\boldsymbol{n})_{u_{j}}\right\rangle & \left\langle\boldsymbol{n}_{u_{i}}, \boldsymbol{n}_{j}\right\rangle \\
1 \leqslant i, j \leqslant s & 1 \leqslant i \leqslant s ; 1 \leqslant j \leqslant r \\
\mathbf{0}_{r \times s} & I_{r}
\end{array}\right) .
\end{aligned}
$$

For $j=1, \ldots, s$ the $\mathrm{j}$-th columns of the two above matrixes satisfy the equation

$$
\widetilde{g}^{j}=\widetilde{g}_{0}^{j}+\sum_{l=1}^{r}\left\langle\boldsymbol{n}_{u_{j}}, \boldsymbol{n}_{l}\right\rangle \widetilde{g}_{0}^{s+l}
$$

It follows that

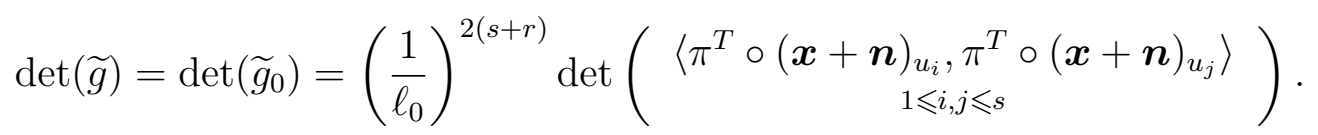

By [11, Proposition 3.2], we have $\pi^{T} \circ(\boldsymbol{x}+\boldsymbol{n})_{u_{i}}=-\sum_{\alpha=1}^{s} \bar{h}_{i}^{\alpha}(\boldsymbol{n}) \boldsymbol{x}_{u_{\alpha}}$, thus

$$
\begin{aligned}
\left\langle\pi^{T} \circ(\boldsymbol{x}+\boldsymbol{n})_{u_{i}}, \pi^{T} \circ(\boldsymbol{x}+\boldsymbol{n})_{u_{j}}\right\rangle & =\left\langle\sum_{\alpha=1}^{s} \bar{h}_{i}^{\alpha}(\boldsymbol{n}) \boldsymbol{x}_{u_{\alpha}}, \sum_{\beta=1}^{s} \bar{h}_{j}^{\beta}(\boldsymbol{n}) \boldsymbol{x}_{u_{\beta}}\right\rangle \\
& =\sum_{\alpha, \beta} \bar{h}_{i}^{\alpha}(\boldsymbol{n}) \bar{h}_{j}^{\beta}(\boldsymbol{n})\left\langle\boldsymbol{x}_{u_{\alpha}}, \boldsymbol{x}_{u_{\beta}}\right\rangle \\
& =\sum_{\alpha, \beta} \bar{h}_{i}^{\alpha}(\boldsymbol{n}) \bar{h}_{j}^{\beta}(\boldsymbol{n}) g_{\alpha \beta} .
\end{aligned}
$$

Finally, by [11, Corollary 3.3], (3.3) and (3.1), we obtain

$$
\operatorname{det}(\widetilde{g})=\left(\frac{1}{\ell_{0}^{s+r}}\right)^{2}\left(K_{h}(\boldsymbol{x}, \boldsymbol{n})\right)^{2} \operatorname{det}\left(g_{\alpha \beta}\right)=\left(\widetilde{K}_{h}(\boldsymbol{x}, \boldsymbol{n})\right)^{2} \operatorname{det}\left(g_{\alpha \beta}\right) .
$$


Definition 3.4. The total absolute horospherical curvature of the immersion $f$ is defined by

$$
\tau_{h}(f)=\frac{1}{\operatorname{vol}\left(S_{+}^{n-1}\right)} \int_{\nu^{1} M} \widetilde{\mathbb{L}}^{*} \sigma .
$$

From Lemma 3.3 we obtain the following formula for $\tau_{h}(f)$ :

$$
\tau_{h}(f)=\frac{1}{\operatorname{vol}\left(S_{+}^{n-1}\right)} \int_{\nu^{1} M}\left|\widetilde{K}_{h}(\boldsymbol{x}, \boldsymbol{n})\right| \mathrm{d} \mu_{\bar{\delta}}(\boldsymbol{x}, \boldsymbol{n}),
$$

where $\bar{\delta}$ denotes the canonical volume density of $\nu^{1} M$. Now we will relate the total absolute horospherical curvature to the critical point of certain height functions.

Definition 3.5. Let $\boldsymbol{v} \in S_{+}^{n-1}$ be a fixed vector. We define the horospherical height function in the $\boldsymbol{v}$ direction as:

$$
h_{\boldsymbol{v}}: M \longrightarrow \mathbb{R}, h_{\boldsymbol{v}}(x)=\langle f(x), \boldsymbol{v}\rangle .
$$

Theorem 3.6. A point $\boldsymbol{x} \in M$ is critical for $h_{\boldsymbol{v}}$ if and only if there is a unit normal $\boldsymbol{n}$ to $M$ at $\boldsymbol{x}$ such that $\widetilde{\mathbb{L}}(\boldsymbol{x}, \boldsymbol{n})=\boldsymbol{v}$. Moreover, $h_{\boldsymbol{v}}$ is a Morse function if and only if $\boldsymbol{v}$ is a regular value of the Horospherical Gauss map.

Let $D \subset S_{+}^{n-1}$ denote the set of regular values of $\widetilde{\mathbb{L}}$. Since $M$ is compact, $D$ is open and, by Sard's theorem, the complement of $D$ in $S_{+}^{n-1}$ has null measure. We define the integer valued map $\kappa: D \rightarrow \mathbb{N}$ by setting

$$
\kappa(\boldsymbol{v})=\text { number of elements of } \widetilde{\mathbb{L}}^{-1}(\boldsymbol{v}),
$$

which turns out to be continuous.

Lemma 3.7. Let $\phi: \nu^{1} M \rightarrow \mathbb{R}$ be a $\mu_{\bar{\delta}}$-integrable function. Then the function $D \ni \boldsymbol{n} \mapsto$

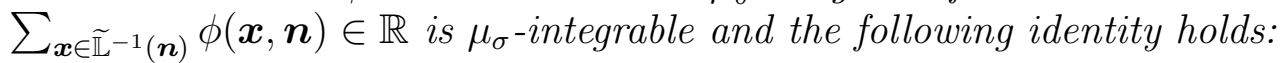

$$
\int_{\nu^{1} M}\left|\widetilde{K}_{h}(\boldsymbol{x}, \boldsymbol{n})\right| \phi(\boldsymbol{x}, \boldsymbol{n}) \mathrm{d} \mu_{\bar{\delta}}(\boldsymbol{x}, \boldsymbol{n})=\int_{D}\left(\sum_{\boldsymbol{x} \in \widetilde{\mathbb{L}}^{-1}(\boldsymbol{n})} \phi(\boldsymbol{x}, \boldsymbol{n})\right) \mathrm{d} \mu_{\bar{\delta}}(\boldsymbol{x}, \boldsymbol{n}) .
$$

Proof. Since $\widetilde{K}_{h}(\boldsymbol{x}, \boldsymbol{n})$ vanishes when $(\boldsymbol{x}, \boldsymbol{n})$ is a critical point of $\widetilde{\mathbb{L}}$ and since the set of regular points of $\widetilde{\mathbb{L}}$ outside $\widetilde{\mathbb{L}}^{-1}(D)$ has null measure we have:

$$
\int_{\nu^{1} M}\left|\widetilde{K}_{h}(\boldsymbol{x}, \boldsymbol{n})\right| \phi(\boldsymbol{x}, \boldsymbol{n}) \mathrm{d} \mu_{\bar{\delta}}(\boldsymbol{x}, \boldsymbol{n})=\int_{\widetilde{\mathbb{L}}^{-1}(D)}\left|\widetilde{K}_{h}(\boldsymbol{x}, \boldsymbol{n})\right| \phi(\boldsymbol{x}, \boldsymbol{n}) \mathrm{d} \mu_{\bar{\delta}}(\boldsymbol{x}, \boldsymbol{n}) .
$$

Observe that the restriction of the horospherical Gauss map to $\widetilde{\mathbb{L}}^{-1}(D)$ is a smooth covering map onto $D$. Hence the conclusion follows by applying Fubini's theorem for covering maps to compute the righthand side of the equality above.

Taking $\phi$ constant and equal to 1 in the above lemma we obtain

Theorem 3.8. Let $f: M^{s} \rightarrow H_{+}^{n}(-1)$ be an immersion of the compact manifold $M$. Then

$$
\tau_{h}(f)=\frac{1}{\operatorname{vol}\left(S_{+}^{n-1}\right)} \int_{D} \kappa(\boldsymbol{v}) \mathrm{d} \mu_{\sigma}(\boldsymbol{v}) .
$$

Recall that the Morse number of a compact manifold $M, \gamma(M)$, is defined as the minimum number of critical points which any Morse function $\varphi: M \rightarrow \mathbb{R}$ can posses. 
TOTAL ABSOLUTE HOROSPHERICAL CURVATURE OF SUBMANIFOLDS IN HYPERBOLIC SPACE 11

Corollary 3.9. Let $f: M^{s} \rightarrow H_{+}^{n}(-1)$ be an immersion of the compact manifold $M$. Then

(1) $\tau_{h}(f) \geqslant \gamma(M) \geqslant 2$;

(2) if $\tau_{h}(f)<3$ then $M$ is homeomorphic to the sphere $S^{s}$.

Proof. Since $M$ is compact, for every $\boldsymbol{v} \in D$, the horospherical height function $h_{\boldsymbol{v}}$ has at least $\gamma(M) \geqslant 2$ critical points, so that $\kappa(\boldsymbol{v}) \geqslant \gamma(M) \geqslant 2$. The proof of (1) follows then from Theorem 3.8. If $\tau_{h}(f)<3$ then $\kappa(\boldsymbol{v})=2$ for some $\boldsymbol{v} \in D$ and therefore $h_{\boldsymbol{v}}$ is a Morse function with exactly two critical points. The proof of the second statement then follows from the well known Reeb's theorem.

When $\tau_{h}(f)=\gamma(M)$ it follows that every non degenerate horospherical height function $h_{\boldsymbol{v}}$ has the minimum number of critical points allowed by the Morse inequalities, ie $f$ is a horo-tight immersion. It follows that $\tau_{h}(f)$ is a hyperbolic invariant when $f$ is a horo-tight immersion.

The relationship between horo-tightness and total absolute horospherical curvature of manifolds deserves further study. For example, we have the following questions:

Question 3.10. How is the geometry of $f(M) \subset H_{+}^{n}(-1)$ if $\tau_{h}(f)=2$ ? Is $\gamma(M)$ the greatest lower bound of $\tau_{h}(f)$ for all smooth immersions $f$ of $M$ into all hyperbolic spaces?

In the following sections we specialize to surfaces and curves in $H_{+}^{3}(-1)$.

\section{Surfaces in $H_{+}^{3}(-1)$}

For an immersion of a closed surface $M$ in Euclidean space, let $K$ denote its Gaussian curvature. Chern and Lashof proved in [4] that

$$
\int_{M}|K| \geqslant 2 \pi(4-\chi(M))
$$

where $\chi(M)$ is the Euler characteristic of $M$. For immersed surfaces in hyperbolic spaces, the inequality (4.1) was expected to hold, according to [16]. However, R. Langevin and G. Solanes in [17] construct examples of surfaces in hyperbolic space which do not satisfy the Chern-Lashof inequality, when the integral is taken with respect to the extrinsic curvature of the surface. In this section we prove that the Chern-Lashof type inequality with respect to the horospherical Gauss-Kronecker curvature holds for surfaces in hyperbolic 3-space.

Theorem 4.1. Let $M^{2}$ be an embedded compact surface in $H_{+}^{3}(-1)$, then

$$
\int_{M}\left|\widetilde{\mathcal{K}}_{h}\right| d \mathfrak{v}_{M} \geqslant 2 \pi(4-\chi(M))
$$

where $\chi(M)$ is the Euler characteristic of $M$ and $d \mathfrak{v}_{M}$ is the volume form of $M$.

Proof. Let $\widetilde{\mathbb{L}}: M^{2} \rightarrow S_{+}^{2}$ denote the horospherical Gauss map of $M^{2} \subset H^{3}$ and let $M^{-}=$ $\left\{p \in M ; \widetilde{\mathcal{K}}_{h}(p)<0\right\}$. We can write

$$
\int_{M}\left|\widetilde{\mathcal{K}}_{h}\right| d \mathfrak{v}_{M}=\int_{M^{+}} \widetilde{\mathcal{K}}_{h} d \mathfrak{v}_{M}-\int_{M^{-}} \widetilde{\mathcal{K}}_{h} d \mathfrak{v}_{M}
$$

and

$$
\int_{M} \widetilde{\mathcal{K}}_{h} d \mathfrak{v}_{M}=\int_{M^{+}} \widetilde{\mathcal{K}}_{h} d \mathfrak{v}_{M}+\int_{M^{-}} \widetilde{\mathcal{K}}_{h} d \mathfrak{v}_{M}
$$


From the horospherical Gauss-Bonnet Theorem and the above equations it follows that

$$
\int_{M}\left|\widetilde{\mathcal{K}}_{h}\right| d \mathfrak{v}_{M}=2 \int_{M^{+}} \widetilde{\mathcal{K}}_{h} d \mathfrak{v}_{M}-2 \pi \chi(M)
$$

The result now follows from Lemma 4.2 below.

Lemma 4.2. Let $M \subset H_{+}^{3}(-1)$ be an embedded compact surface and $M^{+}=\left\{p \in M ; \widetilde{\mathcal{K}}_{h}(p)>\right.$ $0\}$ then

$$
\int_{M^{+}} \widetilde{\mathcal{K}}_{h} d \mathfrak{v}_{M} \geqslant 4 \pi
$$

Proof. Let $M_{0}, M_{1}, M_{2}, M_{2}^{+}$be the subsets of $M$ defined by $M_{0}=\widetilde{\mathcal{K}}_{h}^{-1}(0), M_{1}=\left\{p \in M \backslash M_{0}\right.$; there exists some point $q \in M_{0}$ with $\left.\widetilde{\mathbb{L}}(q)=\widetilde{\mathbb{L}}(p)\right\}, M_{2}=M \backslash\left(M_{0} \cup M_{1}\right)$ and $M_{2}^{+}=M^{+} \cap M_{2}$.

Of course $M_{0}$ is the singular set of $\widetilde{\mathbb{L}}$ so $\widetilde{\mathbb{L}}\left(M_{0}\right)$ has measure zero by Sard's Theorem and also $\widetilde{\mathbb{L}}\left(M_{0}\right) \cup \widetilde{\mathbb{L}}\left(M_{1}\right)$ is a measure zero set. On the other hand, for any $\boldsymbol{v} \in S_{+}^{2} \backslash\left(\widetilde{\mathbb{L}}\left(M_{0}\right) \cup \widetilde{\mathbb{L}}\left(M_{1}\right)\right)$, the horospherical height function $h_{v}$ has at least two critical points: a maximum and a minimum. At such points the horospherical Gauss-Kronecker curvature is positive. The result is then proved since $\widetilde{\mathbb{L}}$ is surjective.

Remark 4.3. If we consider $\mathbb{L}^{-}(p)=f(p)-\mathbb{E}(p)$, we can show that the similar inequality

$$
\int_{M}\left|\widetilde{\mathcal{K}}_{h}^{-}\right| d \mathfrak{v}_{M} \geqslant 2 \pi(4-\chi(M))
$$

holds for another horospherical Gauss-Kronecker curvature $\widetilde{\mathcal{K}}_{h}^{-}(p)=\operatorname{det} \widetilde{S}_{p}^{-}$instead of $\widetilde{\mathcal{K}}_{h}(p)=$ $\widetilde{\mathcal{K}}_{h}^{+}(p)=\operatorname{det} \widetilde{S}_{p}^{+}$. This fact describes the situation in hyperbolic space is quite different from the Euclidean case. The original Chern-Lashof inequality in Euclidean space was given as a corollary of the general codimensional inequality [4]. However, since the unit normal bundle $\nu^{1} M$ has two connected components for a hypersurface, we have the following inequality as a direct consequence of Corollary 3.9:

$$
\frac{1}{4 \pi}\left(\int_{M}\left|\widetilde{\mathcal{K}}_{h}^{+}\right| d \mathfrak{v}_{M}+\int_{M}\left|\widetilde{\mathcal{K}}_{h}^{-}\right| d \mathfrak{v}_{M}\right) \geqslant \gamma(M)
$$

It follows from the Morse relation that $\gamma(M)=4-\chi(M)$ for a closed surafce $M$. Therefore we have an inequlity

$$
\int_{M}\left|\widetilde{\mathcal{K}}_{h}^{+}\right| d \mathfrak{v}_{M}+\int_{M}\left|\widetilde{\mathcal{K}}_{h}^{-}\right| d \mathfrak{v}_{M} \geqslant 4 \pi(4-\chi(M)) .
$$

Although $\mathcal{K}_{h}^{+} \neq \mathcal{K}_{h}^{-}$in general, we have the Chern-Lashof type inequality in Theorem 4.1.

\section{Total absolute horospherical Gaussian Curvature of Curves}

In this section we consider curves in $H_{+}^{3}(-1)$. Let $\gamma: I \longrightarrow H_{+}^{3}(-1)$ be a regular curve. Since $H_{+}^{3}(-1)$ is a Riemannian manifold, we can reparametrise $\boldsymbol{\gamma}$ by the arc-length. Hence, we may assume that $\gamma(s)$ is a unit speed curve. So we have the tangent vector $\boldsymbol{t}(s)=\boldsymbol{\gamma}^{\prime}(s)$ with $\|\boldsymbol{t}(s)\|=1$. In the case when $\left\langle\boldsymbol{t}^{\prime}(s), \boldsymbol{t}^{\prime}(s)\right\rangle \neq-1$, we have a unit vector $\boldsymbol{n}(s)=\frac{\boldsymbol{t}^{\prime}(s)-\boldsymbol{\gamma}(s)}{\left\|\boldsymbol{t}^{\prime}(s)-\gamma(s)\right\|}$. Moreover, define $\boldsymbol{e}(s)=\gamma(s) \wedge \boldsymbol{t}(s) \wedge \boldsymbol{n}(s)$, then we have a pseudo orthonormal frame 
$\{\boldsymbol{\gamma}(s), \boldsymbol{t}(s), \boldsymbol{n}(s), \boldsymbol{e}(s)\}$ of $\mathbb{R}_{1}^{4}$ along $\boldsymbol{\gamma}$. By standard arguments, under the assumption that $\left\langle\boldsymbol{t}^{\prime}(s), \boldsymbol{t}^{\prime}(s)\right\rangle \neq-1$, we have the following Frenet-Serre type formulae:

$$
\left\{\begin{aligned}
\gamma^{\prime}(s) & =\boldsymbol{t}(s) \\
\boldsymbol{t}^{\prime}(s) & =\kappa_{h}(s) \boldsymbol{n}(s)+\gamma(s) \\
\boldsymbol{n}^{\prime}(s) & =-\kappa_{h}(s) \boldsymbol{t}(s)+\tau_{h}(s) \boldsymbol{e}(s) \\
\boldsymbol{e}^{\prime}(s) & =-\tau_{h}(s) \boldsymbol{n}(s)
\end{aligned}\right.
$$

where $\kappa_{h}(s)=\left\|\boldsymbol{t}^{\prime}(s)-\gamma(s)\right\|$ and $\tau_{h}(s)=-\frac{\operatorname{det}\left(\gamma(s), \gamma^{\prime}(s), \gamma^{\prime \prime}(s), \gamma^{\prime \prime \prime}(s)\right)}{\left(\kappa_{h}(s)\right)^{2}}$. We can easily show that the condition $\left\langle\boldsymbol{t}^{\prime}(s), \boldsymbol{t}^{\prime}(s)\right\rangle \neq-1$ is equivalent to the condition $\kappa_{h}(s) \neq 0$. Moreover, we can show that the curve $\gamma(s)$ satisfies the condition $\kappa_{h}(s) \equiv 0$ if and only if there exists a lightlike vector $\boldsymbol{c}$ such that $\gamma(s)-\boldsymbol{c}$ is a geodesic. Such a curve is called an equidistant curve. We can study many properties of hyperbolic space curves by using this fundamental equation. We define $\boldsymbol{N}(s, \theta)=\cos \theta \boldsymbol{n}(s)+\sin \theta \boldsymbol{e}(s)$ as a normal section along $\boldsymbol{\gamma}$. Therefore we have $\mathbb{L}(s, \theta)=\gamma(s)+\boldsymbol{N}(s, \theta)$. By a straightforward calculation, we have

$$
\frac{\partial \mathbb{L}}{\partial \theta}(s, \theta)=\left(1-\kappa_{h}(s)\right) \boldsymbol{t}(s)-\sin \theta \tau_{h}(s) \boldsymbol{n}(s)+\cos \theta \tau_{h}(s) \boldsymbol{e}(s),
$$

and

It follows that we have

$$
\frac{\partial \mathbb{L}}{\partial s}(s, \theta)=-\sin \theta \boldsymbol{n}(s)+\cos \theta \boldsymbol{e}(s)
$$

$$
K_{h}\left(\boldsymbol{N}_{\theta}\right)(\boldsymbol{\gamma}(s))=\left\langle\frac{\partial \mathbb{L}}{\partial \theta}(s, \theta), \boldsymbol{t}(s)\right\rangle=1-\kappa_{h}(s)
$$

so that

$$
\widetilde{K}_{h}\left(\boldsymbol{N}_{\theta}\right)(\boldsymbol{\gamma}(s))=\mathcal{N}_{h}(\boldsymbol{\gamma}(s), \cos \theta \boldsymbol{n}(s)+\sin \theta \boldsymbol{e}(s))^{2}\left(1-\kappa_{h}(s)\right) .
$$

By definition, the total absolute horospherical curvature of $\gamma$ at $s$ is

$$
\widetilde{\kappa}_{h}(s)=\int_{0}^{2 \pi} \mathcal{N}_{h}(\gamma(s), \cos \theta \boldsymbol{n}(s)+\sin \theta \boldsymbol{e}(s))^{2}\left|1-\kappa_{h}(s)\right| d \theta .
$$

By Corollary 2.9, we have the following horospherical Fenchel type theorem:

Theorem 5.1. Let $\boldsymbol{\gamma}: S^{1} \longrightarrow H_{+}^{3}(-1)$ be an embedding. Then we have the following inequality:

$$
\int_{S^{1}} \widetilde{\kappa}_{h} d s \geq 8 \pi
$$

Remark 5.2. We describe here why we say that the above theorem is a Fenchel type theorem. For an embedded curve $\gamma: S^{1} \longrightarrow \mathbb{E}^{3}$ in Euclidean space, Fenchel's theorem asserts that

$$
\int_{S^{1}} \kappa(s) d s \geq 2 \pi
$$

In this case if we consider the canal surface $C M \gamma_{r}$ of $\gamma$ with sufficiently small positive real number $r$, we have a relation

$$
4 \int_{S^{1}} \kappa d s=\int_{C M \gamma_{r}}|K| d \mathfrak{v}_{C M \gamma_{r}}
$$


So the Fenchel's theorem follows from the Euclidean Chern-Lashof inequality. However for the horospherical curvature, we have no such a relation.

We can also show the following horospherical Fary-Milnor type theorem.

Theorem 5.3. Let $\gamma: S^{1} \longrightarrow H_{+}^{3}(-1)$ be an embedding. If $\boldsymbol{\gamma}$ is knotted, then

$$
\int_{S^{1}} \widetilde{\kappa}_{h} d s \geq 16 \pi
$$

Before we start to give the proof, we need another interpretation of the total absolute horospherical curvature of a surface $f: M \longrightarrow H_{+}^{3}(-1)$.

We now consider an integer valued function $\eta: S_{+}^{2} \backslash C \longrightarrow \mathbb{N}$ defined by

$$
\eta(\boldsymbol{v})=\text { the number of } \widetilde{\mathbb{L}}^{-1}(v),
$$

where $C=\widetilde{\mathbb{L}}\left(\widetilde{\mathcal{K}}_{h}^{-1}(0)\right)$ (i.e., the critical value set of $\widetilde{\mathbb{L}}$.). By Sard's Theorem, $C$ has measure zero, so that $\eta$ is a well-defined continuous function almost everywhere in $S^{2}$. Any point $\boldsymbol{v} \in S_{+}^{2}$ has a neighbourhood $U$ on which $\eta$ has a constant value $m$ and such that $\widetilde{\mathbb{L}}^{-1}(U) \subset M$ is the disjoint union of $m$ open sets $V_{1}, \ldots, V_{m}$ on each of which $\widetilde{\mathbb{L}}: V_{\alpha} \longrightarrow U$ is a diffeomorphism. Since $\widetilde{\mathbb{L}}^{*} d \mathfrak{v}_{S_{+}^{2}}=\widetilde{\mathcal{K}}_{h} d \mathfrak{v}_{M}$, we have

$$
\int_{V_{\alpha}} \widetilde{\mathcal{K}}_{h} d \mathfrak{v}_{M}= \begin{cases}\int_{U} d \mathfrak{v}_{S_{+}^{2}} & \text { if } \widetilde{\mathcal{K}}_{h}>0 \\ -\int_{U} d \mathfrak{v}_{S_{+}^{2}} & \text { if } \widetilde{\mathcal{K}}_{h}<0 .\end{cases}
$$

So

From this we see that

$$
\int_{V_{\alpha}}\left|\widetilde{\mathcal{K}}_{h}\right| d \mathfrak{v}_{M}=\int_{U} d \mathfrak{v}_{S_{+}^{2}}
$$

$$
\int_{M}\left|\widetilde{\mathcal{K}}_{h}\right| d \mathfrak{v}_{M}=\int_{S_{+}^{2} \backslash C} \eta d \mathfrak{v}_{S_{+}^{2}}
$$

We also need some definitions. If the horosphere is defined by $H_{+}^{3}(-1) \cap H P(\boldsymbol{v}, c)$ for a lightlike vector $\boldsymbol{v}$, we say that the center direction of the horosphere is $\boldsymbol{v}$. Moreover if we have two points $\boldsymbol{x}_{1}, \boldsymbol{x}_{2} \in H_{+}^{3}(-1) \cap H P(\boldsymbol{v}, c)$, we have a lightlike affine plane

$$
L P\left(\boldsymbol{x}_{1}, \boldsymbol{x}_{2} ; \boldsymbol{v}\right)=\left\{\lambda \boldsymbol{x}_{1}+(1-\lambda) \boldsymbol{x}_{2}+\mu \boldsymbol{v} \mid \lambda, \mu \in \mathbb{R}\right\} \subset H P(\boldsymbol{v}, c) .
$$

Then we have a horocycle defined by $H_{+}^{3}(-1) \cap L P\left(\boldsymbol{x}_{1}, \boldsymbol{x}_{2} ; \boldsymbol{v}\right)$. We call it the horocycle joining $\boldsymbol{x}_{1}, \boldsymbol{x}_{2}$ with the center direction $\boldsymbol{v}$. Under these preparations, we can prove Theorem 5.3.

Proof. Suppose that

$$
\int_{S^{1}} \widetilde{\kappa}_{h} d s<16 \pi
$$

Then we have

$$
\int_{S_{+}^{2}} \eta d \mathfrak{v}_{S_{+}^{2}}<16 \pi
$$

This means that some point $\boldsymbol{v} \in S_{+}^{2}$ is the image of $\widetilde{\mathbb{L}}$ at most 3 points of $M=\nu_{1}\left(\gamma\left(S^{1}\right)\right)$. Since the hyperbolic Gauss indicatrix of $M$ is

$$
\mathbb{L}(s, \theta)=\gamma(s)+\boldsymbol{N}(s, \theta)=\boldsymbol{\gamma}(s)+(\cos \theta \boldsymbol{n}(s)+\sin \theta \boldsymbol{e}(s)),
$$


the above condtion is equivalent to saying that $\boldsymbol{t}(s)=\boldsymbol{\gamma}^{\prime}(s)$ is pseudo-perpendicular to $\boldsymbol{v}$ for at most 3 values of $s$. Since

$$
\left\langle\boldsymbol{v}, \boldsymbol{\gamma}^{\prime}(s)\right\rangle=\frac{d}{d s}\langle\boldsymbol{v}, \boldsymbol{\gamma}(s)\rangle,
$$

the hyperbolic height function from $\widetilde{h}_{v}(s)=\langle v, \gamma(s)\rangle$ of $\boldsymbol{\gamma}$ has at most 3 critical points. Since the number of relative maxima or minima of the hyperbolic height function is even, there must be just one of each. Therefore the curve $\gamma$ must consist of two arcs joining the lowest and highest points, each arc having monotonically increasing height relative to $\boldsymbol{v}$ direction. Since the level surface of the hyperbolic height function is a horosphere, each horosphere $H_{+}^{3}(-1) \cap H P(\boldsymbol{v}, c)$ with the center direction $\boldsymbol{v}$ between these lowest and highest points intersects the curve in 2 points. Joining each such pair by the horocycle with the center direction $\boldsymbol{v}$, we obtain an embedded disc whose boundary is the curve $\boldsymbol{\gamma}$. Therefore $\boldsymbol{\gamma}$ is unknotted. This completes the proof.

\section{REFERENCES}

[1] R. L. Bryant, Surfaces of mean curvature one in hyperbolyc space, Théorie de variétés minimales et applications (Palaiseau, 1983-1984), Astèrisque No. 154-155, (1987), 12, 321-347, 353 (1988).

[2] T. E. Cecil and P. J. Ryan, Tight ant taut immersions into hyperbolic space, J. London Math. Soc., 19 (1979), 561-572.

[3] T. E. Cecil and P. J. Ryan, Distance functions and umbilic submanifolds of hyperbolic space, Nagoya Math. J., 74 (1979), 67-75.

[4] S. S. Chern and R. K. Lashof, On the total absolute curvature of immersed manifolds I, Amer. J. Math. 79 (1957) 306-318.

[5] C. L. Epstein, The hyperbolic Gauss map and quasiconformal reflections. J. Reine Angew. Math., 372 (1986), 96-135

[6] S. Izumiya, D. Pei and T. Sano, Singularities of hyperbolic Gauss maps, Proc. London Math. Soc. 86 (2003) 485-512. 65 (2004) 107-123.

[7] S. Izumiya, D. Pei and T. Sano, Horospherical surfaces of curves in Hyperbolic space, Publ. Math. Debrecen, 64 (2004), 1-13.

[8] S. Izumiya, D. Pei and M. Takahashi, Curves and surfaces in hyperbolic space, Geomtric singularity theory, Banach Center Publications,

[9] S. Izumiya, D-H. Pei and M. Takahashi, Singularities of evolutes of hypersurfaces in hyperbolic space, Proceedings of the Edinburgh Mathematical Society, 47 (2004), 131-153. 65 (2004) 107-123.

[10] S. Izumiya, D. Pei, M. C. Romero-Fuster and M. Takahashi, On the horospherical ridges of submanifolds of codimension 2 in Hyperbolic n-space, Bull. Braz. Math. Soc. 35 (2) (2004), 177-198.

[11] S. Izumiya, D. Pei, M. D. R. Fuster, and M. Takahashi, The horospherical geometry of submanifolds in hyperbolic space, J. London Math. Soc., (2) 71 (2005) 779-800.

[12] S. Izumiya, and M. D. R. Fuster, The horospherical Gauss-Bonnet type theorem in hyperbolic space, Journal of Mathematical Society of Japan 58 (2006) 965-984.

[13] S. Izumiya, D. Pei and M. C. Romero-Fuster, The horospherical geometry of surfaces in Hyperbolic 4-space, Israel Journal of Mathematics, 154 (2006), 361-379.

[14] T. Kobayashi, Null varieties for convex domains (Japanese). Reports on unitary representation seminar 6 (1986), 1-18

[15] T. Kobayashi, Asymptotic behaviour of the null variety for a convex domain in a non-positively curved space form. Journal of the Faculty of Science, University of Tokyo, 36 (1989), 389-478

[16] B. A. Salemi and T. J. Willmore, Total absolute curvature of immersed manifolds, J. London Math. Soc., 41 (1966) 153-160.

[17] R. Langevin and G. Solanes, On bounds for total absolute curvature of surfaces in hyperbolic 3-space, C. R. Acad. Sci. Paris, Ser. I 336 (2003). 
Universidade Federal do Espírito Santo, Departamento de Matemática, Av. Fernando Ferrari, 514, Goiabeiras, 29075-910, Vitória, ES, BraZil.

Department of Mathematics, Hokkaido University, Sapporo 060-0810, Japan.

Instituto de Ciências Matemáticas e de Computação, Universidade de São Paulo, Departamento de Matemática, Caixa Postal 668, 13560-970, São Carlos, SP, Brazil.

E-mail address, Marcelo Buosi: pmarpceplo@yahoo.com.br

E-mail address, Shyuichi Izumiya: izumiya@math.sci.hokudai.ac.jp

E-mail address, Maria Aparecida Soares Ruas: maasruas@icmc.usp.br 